\title{
Levels of Salivary Sialic Acid in Children with Autism Spectrum Disorder; Could It Be Related to Stereotypes and Hyperactivity?
}

\author{
Esra Demirci ${ }^{1}$, Yunus Guler', Sevgi Ozmen ${ }^{1}$, Mehmet Canpolat ${ }^{3}$, Sefer Kumandas ${ }^{3}$ \\ ${ }^{1}$ Department of Child and Adolescent Psychiatry, ${ }^{2}$ Department of Pediatrics, ${ }^{3}$ Department of Pediatric Neurology, Erciyes University School \\ of Medicine, Kayseri, Turkey
}

\begin{abstract}
Objective: Sialic acid (Sia) is an essential nutrient for brain development, learning, memory and cognition and plays a role in neurodevelopment of infants. The aim of this study was to determine whether Sia levels are significantly associated with the autism spectrum disorder (ASD).

Methods: Forty-six ASD children and 30 typically developing children aged 3 to 10 years were included in the study. Behavioral symptoms in ASD children was assessed by the Autism Behavior Checklist (AuBC), the Childhood Autism Rating Scale, and the Aberrant Behavior Checklist (ABC). After the collection of saliva samples, the supernatant was separated. All the samples kept at $-80^{\circ} \mathrm{C}$ until Sia analysis was done.

Results: Sia level was found to be significantly lower in the ASD group when compared to healthy controls $(p=0.013)$. There was no correlation between severity of ASD and salivary Sia levels. We found a negative correlation between AuBC scores and Sia levels and a negative correlation in both ABC Stereotypic Behavior and Hyperactivity/Noncompliance subscales with Sia levels in ASD group.

Conclusion: The obtained data indicate that Sia levels could have an effect on autism-like behaviors, particularly on stereotypes and hyperactivity.
\end{abstract}

KEY WORDS: Saliva; Sialic acid; Child; Autism spectrum disorder; Stereotypes.

\section{INTRODUCTION}

Autism spectrum disorder (ASD) is a neurodevelopmental disorder that begins early in childhood and is characterized by social deficits, impaired communication, and repetitive stereotypic behaviors. The prevalence of ASD is currently estimated to be one in every 68 children. ${ }^{1)}$ Despite intense research efforts over the past decade, the etiology of idiopathic ASD is stil unknown whereas syndromic ASD is secondary to a primary condition caused by a single gene mutation, for example fragile $X$ syndrome. ${ }^{2)}$ Also, researchers suggested that the place alterations in developmental transcriptional regulation, brain growth, changes in the excitatory/inhibitory

Received: December 11, 2018 / Revised: January 24, 2019

Accepted: January 25, 2019

Address for correspondence: Esra Demirci

Department of Child and Adolescent Psychiatry, Erciyes

University School of Medicine, 38039-Kayseri, Turkey

E-mail: esra_z_d_r@hotmail.com

ORCID: https://orcid.org/0000-0002-8424-4947 balance of the neural network, and abnormalities in neural plasticity might have a role in pathogenesis. ${ }^{3)}$

Sialic acid (Sia) is an essential nutrient for brain development and cognition and plays a role in neurodevelopment of infants. ${ }^{4)}$ In mammals, the highest concentration of Sia is found in the central nervous system, mostly as structural and functional part in gangliosides and glycoproteins. ${ }^{5)}$ Interruption of Sia biosynthesis is embryonic lethal in mice, moreover subtle mutations in Sia metabolism result in a variety of diseases in humans. ${ }^{6}$ It was reported that dietary Sia supplementation improves learning and memory in piglets. ${ }^{7)}$ Also, Sia can be synthesized into polysialic acid glycan (polySia), which associates with neural cell adhesion molecules (NCAM) to form polySia-NCAM, which in turn impacts molecular interactions during synaptic plasticity and neural development. ${ }^{4)}$

In the most of the studies, autistic rodent models are typically used for functional studies because of the difficulties in obtaining brain tissue samples for studies. In

(ㄷ) This is an Open-Access article distributed under the terms of the Creative Commons Attribution Non-Commercial License (http://creativecommons.org/licenses/by-nc/4.0) which permits unrestricted non-commercial use, distribution, and reproduction in any medium, provided the original work is properly cited. 
these studies, Calandreau et al. ${ }^{8)}$ found a direct correlation between polySia and ASD-like behaviors and reported that mice have deficient in ST8SIAll exhibited reduced social motivation, increased aggression and hyperactivity. Variants of ST8SIA2, has a role in polySia synthesis, have also been associated with ASD. ${ }^{9)}$ Especially, two intronic single nucleotide polymorphisms of ST8SIA2 (rs2168351 and rs3784730) were associated with ASD. ${ }^{10)}$

Also, given the relevant literature, it was reported that gangliosides and glycoproteins, containing Sia in their structure, may play a role in the etiology of ASD, ${ }^{5,11)}$ In addition, brain-derived neurotrophic factor (BDNF) level, which are also reported to be a predictor of autism, ${ }^{12)}$ is regulated by polySia. ${ }^{13)}$ However, limited information is available about the relationship between Sia and ASD in children. To our knowledge, there was only one study investigating the relationship between plasma Sia levels and the pathogenesis of ASD in humans. They found that the level of plasma Sia in the control group higher than that in the children with ASD. ${ }^{14)}$ Also, in 2014, a child diagnosed with ASD and epilepsy was found to have a heterozygous $520-k b$ deletion on chromosome 15q26.1, where ST8SIA2 is located. ${ }^{15)}$ This case, also supports the evidences that the ST8SIA2 haploinsufficiency play a role in neurobehavioral phenotypes. Altogether, further studies are needed to understand the relationship between Sia and ASD.

There are some advantages of using saliva instead of blood in clinical practice, as non-invasive saliva collection is devoid of stress, with no risk of needlestick injuries and easy to perform, and self-collection of saliva sample is possible after simple instructions. ${ }^{16)}$ Also, it was reported that saliva could be a marker for Sia availability, with implications for the accretion of Sia in brain gangliosides. ${ }^{17)}$ Considering the advantages of working with saliva as well as the lack of studies in children, the current study sought to investigate the association between salivary Sia and the ASD in children.

\section{METHODS}

\section{Participants}

The case-control study included 46 ASD children and 30 typically developing children between 3 and 10 years of age. Twenty-eight patients consisted of newly diagnosed children. Also, only 18 patients in the ASD group were receiving risperidone treatment (duration of treatment, 2-6 months; mean \pm standard deviation [SD], 0.32 $\pm 0.013 \mathrm{mg} /$ day) and there were no drug use except those 18 patients. Patients and controls were selected after performing physical, psychiatric and neurologic examinations, routine biochemical tests, complete blood counts, and thyroid function tests. All autistic children were diagnosed by an experienced child and adolescent psychiatrist based on the criteria of the Diagnostic and Statistical Manual of Mental Disorders, 5th edition (DSM-5) ${ }^{18)}$ and by an experienced child neurologist. Also, the control group showed neither clinical nor laboratory signs of any disorders, and were not related to the ASD group.

Evaluation of behavioral symptoms in ASD children based on clinical history, clinical examination and neuropsychiatric assessment, the Autism Behavior Checklist (AuBC), the Childhood Autism Rating Scale (CARS) and the Aberrant Behavior Checklist (ABC). The AuBC was developed to assess and screen for autism and to evaluate the severity of autism symptoms and divided into five categories: sensory, relating (social skills), body and object use, language, social and self help. ${ }^{19)}$ The CARS is a 15 item behavioral rating scale developed to identify children with autism and to categorize these behaviors from mild to moderate to severe. ${ }^{20)}$ The total CARS score may range from a low of 15 to a high of 60 . The score represents placement on a continuum: the lower the score, the fewer autistic behaviors the child exhibits; the higher the score, the more autistic behaviors the child exhibits. The $A B C$ is a scales designed to measure psychiatric symptoms and behavioral disturbance exhibited by individuals with intellectual and developmental disabilities across 5 domains: irritability/agitation/crying; lethargy/social withdrawal; stereotypic behavior; hyperactivity/noncompliance; and inappropriate speech. ${ }^{21)}$

The exclusion criteria were fragile $X$ syndrome, any other genetic disorders, psychiatric disorders especially attention deficit hyperactivity disorder and tic disorder, mental retardation, history of infection in two weeks and intake of any medicine in two days for both groups. Participants with poor oral hygiene were also excluded from the study.

The reason for the fact that Sia is an essential nutrient, participant's nutrition-related characteristics was questioned in detail in the sociodemographic forms.

This study was approved by the local ethical committee 
of the university (2015/579). In addition, written informed consents were obtained from the parents of the children who participated in the study.

\section{Measurement of Sialic Acid}

Parents were informed that children should not have food and drinks 2 hours before the saliva collection. All samples $(1.5$ to $2 \mathrm{ml})$ of the residual whole saliva were collected into plastic tubes placed on ice by spitting method, between 8:00 AM and 9:00 AM to minimize the influence of the circadian rhythms, and centrifuged $(3,000 \times g$; 15 minutes) and the supernatant was separated. All samples kept at $-80^{\circ} \mathrm{C}$ until Sia analysis was done. Sialic acid assay kit is used to quantitative determination of Sia. The levels of Sia were measured in accordance with the manufacturer's instructions. Ninety-three microlitter assay buffer, $1 \mu \mathrm{l}$ dye reagent and $1 \mu$ enzyme were placed in the standard solutions prepared for calibration and incubated in the cold cupboard. After standing at room temperature for 60 minutes, the optical density was read at $570 \mathrm{~nm}$ on the epoch microplate spectrophotomete.

\section{Statistical Analysis}

The study data were analyzed with the IBM SPSS Statistics ver. 21.0 (IBM Corp., Armonk, NY, USA) and SigmaStat 3.5 statistical software packages (Systat Software, Inc., San Jose, CA, USA). The variables were expressed as number, percentage, mean and SD by using descriptive

Table 1. Characteristics of patients and healthy controls

\begin{tabular}{lcc}
\hline \multicolumn{1}{c}{ Characteristic } & ASD group & Control group \\
\hline Age $(y r)$ & $5.50 \pm 2.05$ & $5.35 \pm 2.15$ \\
CARS, total score & $49.96 \pm 15.04$ & $15.03 \pm 5.35$ \\
AuBC & & \\
$\quad$ Total score & $83.43 \pm 37.21$ & $12.56 \pm 8.05$ \\
$\quad$ Body and object use behaviors & $20.13 \pm 14.27$ & $3.12 \pm 4.53$ \\
ABC & & \\
Total scores & $76.61 \pm 29.50$ & $15.27 \pm 3.15$ \\
Irritability factor & $25.20 \pm 7.90$ & $13.51 \pm 10.35$ \\
Lethargy factor & $9.50 \pm 6.35$ & 0 \\
Stereotypic behavior factor & $8.02 \pm 4.20$ & $1.45 \pm 0.55$ \\
Hyperactivity/noncompliance & $24.20 \pm 14.10$ & $5.43 \pm 4.25$ \\
$\quad$ factor & & 0 \\
Inappropriate speech factor & $5.34 \pm 2.16$ & \\
\hline
\end{tabular}

Values are presented as mean \pm standard deviation.

ASD, autism spectrum disorder; CARS, the Childhood Autism Rating Scale; AuBC, Autism Behavior Checklist; ABC, the Aberrant Behavior Checklist.

Independent $t$ test was used; $p>0.05$ for all comparisions. statistical tests. The Shapiro-Wilk test was used to evaluate whether the data were normally distributed. Independent $t$ test was used to compare scale scores between two groups. One-way ANOVA, general linear models and Bonferroni test were used for the comparison of inter groups and comparisons were adjusted by gender. The Pearson correlation test was used to determine the correlations among the variables. Statistical significance was set at $p<0.05$.

\section{RESULTS}

The ASD group had 46 children (36 males, 10 females, and a male to female ratio 3.6:1); the mean age was $5.50 \pm$ 2.05 years old. The control group included 30 children (21 males, 9 females, and a male to female ratio 2.3:1); the mean age was $5.35 \pm 2.15$ years old. No significant difference was found between the patients and healthy children in terms of age $(p>0.05)$ and gender $(p>0.05)$. CARS score was $49.96 \pm 15.04$ in the ASD group. Fifty percent $(n=23)$ of the patients were mild-moderate ASD and $50 \%(n=23)$ were severe ASD. AuBC and ABC scores were also given in Table 1.

It was found that the duration of breastfeeding is shorter and age of the transition to feeding with solid food is older in ASD group compared to controls. It was seen gastrointestinal system pathologies such as lactose intolerance and inflammatory bowel syndrome more frequent, and the diversity of food consumption is limited in ASD group as expected (Table 2).

Table 2. Comparison of feeding forms and characteristics of both groups

\begin{tabular}{lcc}
\hline \multicolumn{1}{c}{ Variable } & $\begin{array}{c}\text { ASD group } \\
(\mathrm{n}=46)\end{array}$ & $\begin{array}{c}\text { Control } \\
\text { group } \\
(\mathrm{n}=30)\end{array}$ \\
\hline Duration of breastfeeding (mo) & $4.3 \pm 2.5$ & $13.3 \pm 3.2$ \\
Transition to feeding with solid food (mo) & $11.5 \pm 3.2$ & $7.6 \pm 4.4$ \\
Suffer from colic & $19(41.3)$ & $6(20.0)$ \\
Suffer from reflux and vomiting & $13(28.2)$ & $3(10.0)$ \\
Selective and restricted nutrition & $37(80.4)$ & $7(23.3)$ \\
Constipation & $3(6.5)$ & $3(10.0)$ \\
Lactose intolerance & $7(15.2)$ & $0(0)$ \\
Inflammatory bowel syndrome & $4(8.6)$ & $0(0)$ \\
Other gastrointestinal system pathologies & $1(2.1)$ & $0(0)$ \\
\hline
\end{tabular}

Values are presented as mean \pm standard deviation or number (\%). ASD, autism spectrum disorder.

Descriptive statistical tests was used. 
Salivary Sia level was found to be significantly lower in the ASD group $(0.101 \pm 0.077 \mathrm{mmol} / \mathrm{L})$ when compared to the healthy controls $(0.160 \pm 0.097 \mathrm{mmol} / \mathrm{L})(p=$ 0.007). The level of Sia in the control group was higher than that in the ASD groups with and without medication (Table 3, Fig. 1). There were no significant differences in the levels of Sia when comparing the ASD groups with and without medication (Table 3). All comparisons were adjusted by gender, levels of Sia was not affected by gender in all groups.

To check the reliability of results with subsequent samples, second saliva samples were taken two weeks after the first samples were taken from 25 participants (15 ASD, 10 controls), selected randomly, and no difference was detected between the first and second samples in both ASD and the control groups ( $p=0.87$ and 0.93 , respectively).

After assessing the severity of ASD in children with CARS, we did not find any correlation between the se-

Table 3. Comparison of salivary sialic acid (Sia) levels between groups

\begin{tabular}{cc}
\hline \multicolumn{1}{c}{ Group } & Sia level $(\mathrm{mmol} / \mathrm{L})$ \\
\hline ASD group & \\
Without medication $(\mathrm{n}=28)$ & $0.102 \pm 0.062^{*}$ \\
With medication $(\mathrm{n}=18)$ & $0.100 \pm 0.099^{*}$ \\
Healthy controls $(\mathrm{n}=30)$ & $0.160 \pm 0.097$ \\
\hline
\end{tabular}

Values are presented as mean \pm standard deviation.

General linear models and Bonferroni test were used; adjusted by sex; not effected from sex.

*Lower than healthy controls $(p=0.027), \eta^{2}=0.101$. verity of ASD and salivary Sia levels $(r=0.276, p=0.123)$. However, there was a negative correlation between AuBC total scores and Sia levels ( $r=-0.383, p=0.013$ ) (Table 4). As an interesting result; we found a negative correlation between AuBC body and object use behaviors subscales and Sia levels $(r=-0.476, p<0.001)$. Likewise, we found negative correlation between $A B C$ stereotypic behavior subscales and Sia levels, and we also found a negative correlation between hyperactivity/noncompliance subscales and Sia levels in children with ASD $(r=-0.293$, $p=0.004$ and $r=-0.276, p=0.026$, respectively) (Table 4).

Receiver operating characteristic (ROC) curve analysis was used to evaluate the predictive power of Sia levels to distinguish between ASD children and controls. The analysis showed that Sia has stronger diagnostic value with high sensitivity $(76.3 \%)$ and specificity $(78.0 \%)$ to distinguish healthy children than to diagnosed ASD (Fig. 2) with an area under the ROC curve value of 0.751 .

Table 4. Correlations between sialic acid (Sia) levels and scales scores

\begin{tabular}{lc}
\hline \multicolumn{1}{c}{ Scale } & Sia level $(\mathrm{mmol} / \mathrm{L})$ \\
\hline CARS, total score & $\mathrm{r}=0.276, p=0.123$ \\
AuBC & $\mathrm{r}=-0.383, p=0.013$ \\
Total scores & $\mathrm{r}=-0.476, p<0.001$ \\
Body and object use behaviors & \\
subscales & \\
ABC & $\mathrm{r}=-0.293, p=0.004$ \\
Stereotypic behavior subscales & $\mathrm{r}=-0.276, p=0.026$ \\
Hyperactivity/Non compliance & \\
subscales &
\end{tabular}

A

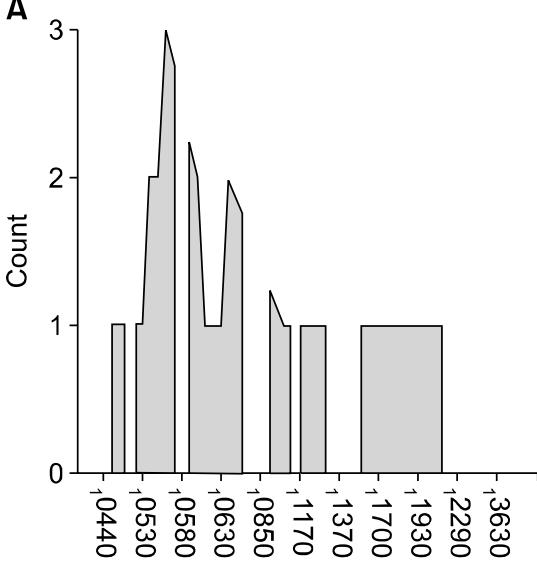

B

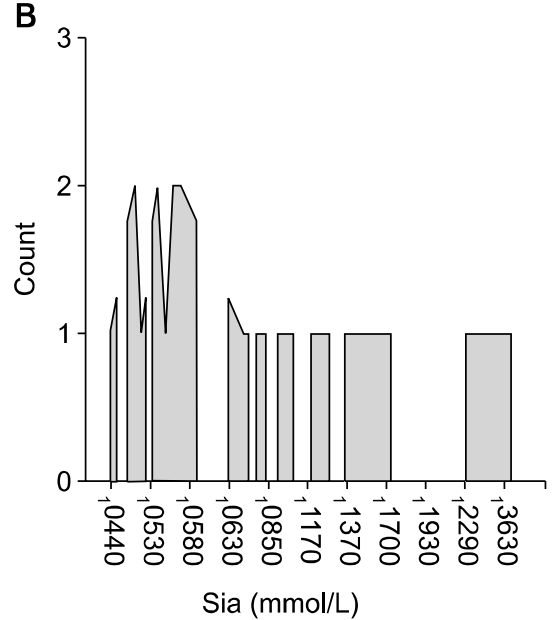

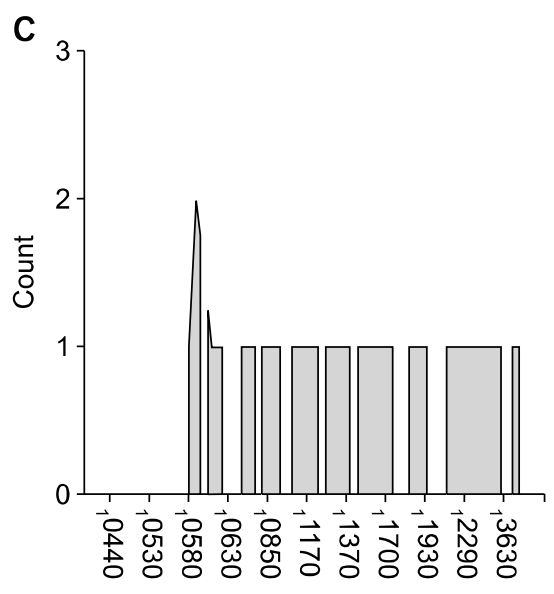

Fig. 1. Comparison of salivary sialic acid (Sia) levels between groups. (A) Autism spectrum disorder (ASD) without medication. (B) ASD with medication. (C) Healthy controls. 

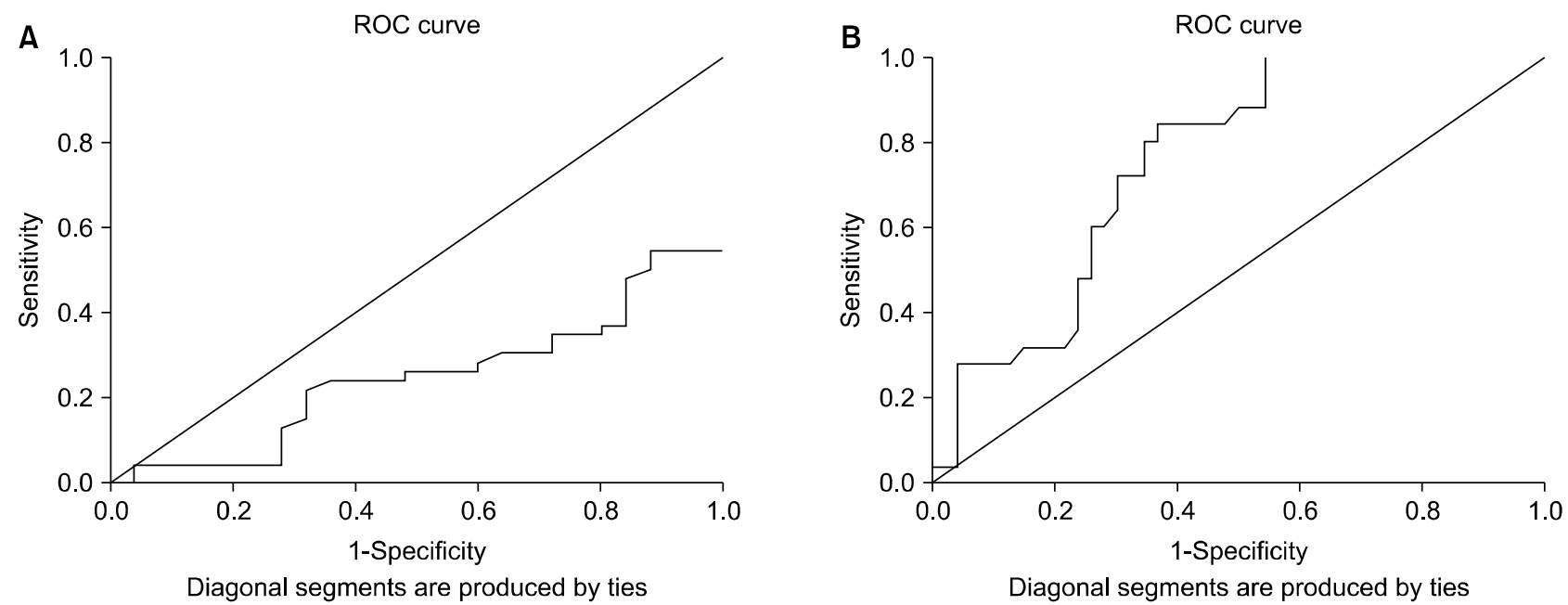

Fig. 2. Receiver operating characteristic (ROC) curve. (A) ROC curve of sialic acid (Sia) levels for autism spectrum disorder group. (B) ROC curve of Sia levels for controls.

\section{DISCUSSION}

The main finding of the present study was that the level of Sia in the ASD group with or without medication was lower than that in the control group. There is only one study in the literature that assessed Sia levels in children with ASD. In this study, 82 children with ASD were evaluated and plasma Sia levels were found to be statistically lower than healthy controls. Also, a negative correlation was found between the severity of ASD and Sia levels, and it was stated that Sia levels could be a predictor for ASD. Unlike this study, we did not find any correlations between CARS scores and Sia levels in our study. We have also found that the Sia levels could be acceptable as a marker of being healthy. With it, as an interesting result, there was a negative correlation between AuBC scores and Sia levels, and a negative correlation between AuBC body and object use behaviors subscales and Sia levels. We also found a negative correlation between $A B C$ stereotypic behavior subscales and Sia levels, and a negative correlation between hyperactivity/noncompliance subscales and Sia levels in the children with ASD. These results in our study can be interpreted as that salivary Sia levels may be more related to autistic symptoms and especially stereotypes and hyperactivity/noncompliance than the ASD severity. PolySia draws attention when the studies that can support this hypothesis in the literature are evaluated. In analytical studies of polySia-impaired mice and polySia-cleaving enzyme-injected mice, it has been shown that polySia have effects on learning and memory, circadian rhythms, emotion and social interactions, and the anatomical changes, including reduction of interneurons in prefrontal cortex, enlargement of ventricles, reduction of thalamus, and hypoplasia of specific axon tracts, are considered to be involved in the abnormality of behavior in polySia-impaired mice. ${ }^{5,22)}$ Also, the intracellular concentration of Sia appears to play a key role in regulating the polysialylation of NCAM and polySia has an essential role in regulating NCAM-dependent developmental processes that require plasticity, that is, cell migration, axon guidance and synapse formation. ${ }^{23)}$ PolySiapositive neurons are present in different cortical areas, including the prefrontal cortex, piriform cortex, hippocampus, septum and amygdala. ${ }^{5,24)}$ These cells are inhibitory interneurons characterized by the presence of the GABAergic marker glutamic acid decarboxylase. PolySianegative interneurons receive more synaptic input than their polySia-positive counterparts and show induced dendritic arborization and spine numbers. These morphological features are consistent with the notion that polySia is a negative regulator of interneuron connectivity and may support plasticity of inhibitory cortical networks. ${ }^{24,25)}$ On this point, it is knowns that there are evidence of abnormalities in GABAergic neurons and synapses in neurodevelopmental disorders characterised by a shared symptomatology of ASD symptoms and GABAergic inhibition is implicated in several developmental disorders such as ASD. ${ }^{26)}$ Dysfunction in GABA signalling mediates autism-like 
stereotypies and Rett syndrome phenotypes. ${ }^{27)}$ Furthermore, it was reported that possible GABAergic dysfunction within corticostriatal pathways in children with primary complex motor stereotypies. ${ }^{28)}$ In addition, there is evidence that polySia is effective in the regulation of molecules such as BDNF and dopamine. ${ }^{29)}$ It is known that both BDNF and dopamine can reciprocally potentiate each other and have an effect on the pathogenesis of hyperactivity, stereotypes and autistic like behaviours. ${ }^{29)}$ Also, increased BDNF expression in the striatum associated with the enrichment-related prevention of stereotyped behavior. ${ }^{30)}$ We found no statistically significant effect of Sia levels on gender in our study in accordance with the literature. $^{7)}$

It is known that the Sia is an essential nutrient and Human milk is noteworthy in containing exceptionally high levels of Sia-glycoconjugates. ${ }^{4)}$ Dietary sialyllactose influences Sia concentrations in the prefrontal cortex, hippocampus and magnetic resonance imaging measures in corpus callosum of young pigs. ${ }^{31)}$ Findings from studies with piglets also suggests that supplementation with bovine lactoferrin leads to increased hippocampal mRNA, protein concentrations of BDNF and polySia, and also facilitates enteric nervous system development by upregulating duodenal mRNA expression of BDNF. ${ }^{32,33)}$ Moreover, some studies have demonstrated a significant protective effect of breastfeeding on the development of ASD. ${ }^{34,35)}$ and brain ganglioside and glycoprotein Sia levels are higher in breastfed infants compared to formula-fed infants. ${ }^{36)}$ In addition, it was known that the gastrointestinal symptoms and disorders are associated with autism. ${ }^{37)}$ In our study, we found that the duration of breastfeeding is shorter, age of the transition to feeding with solid food is older in ASD group compared to controls. Also, it was seen gastrointestinal system pathologies such as lactose intolerance and inflammatory bowel syndrome more frequent, and the diversity of food consumption is limited in ASD group. Shorter breastfeeding duration, frequent occurrence of nutritional problems in ASD could be a cause of increased Sia levels and Sia's essential replacement could decrease ASD like behavior including stereotypes and hyperactivity.

As a conclusion, it is clear that there is a need for wide-ranging studies to determine whether Sia levels, associated with polysialia levels, have an effect on autism-like behaviors, particularly on stereotypes. Also, pro- spective longitudinal researches could be guided to evaluate whether or not the Sia's essential replacement will have an effect on ASD like behavior including stereotypes and hyperactivity.

There are some limitations to the current study. We could not measure the level of polySia and ST8SIA2 in ASD and controls. Also, Sia is an important nutrient for nervous system development; however, we could not assess the oral intake levels/per day of the Sia. Thus, an increased number of samples from children may be needed in a follow-up study.

\section{- Acknowledgments}

Because of the contributions she made to our study, we thank to Serife Sacmacı from the Department of Chemistry, Erciyes University Science Faculty and we also thank to all assistant doctors of Department of Child and Adolescent Psychiatry, Erciyes University Medicine Faculty, who have contributions in assessments with CARS.

\section{- Conflicts of Interest}

No potential conflict of interest relevant to this article was reported.

\section{REFERENCES}

1. Developmental Disabilities Monitoring Network Surveillance Year 2010 Principal Investigators; Centers for Disease Control and Prevention (CDC). Prevalence of autism spectrum disorder among children aged 8 years - autism and developmental disabilities monitoring network, 11 sites, United States, 2010. MMWR Surveill Summ 2014;63:1-21.

2. Geschwind DH, State MW. Gene hunting in autism spectrum disorder: on the path to precision medicine. Lancet Neurol 2015;14:1109-1120.

3. Dwyer CA, Esko JD. Glycan susceptibility factors in autism spectrum disorders. Mol Aspects Med 2016;51:104-114.

4. Wang B. Molecular mechanism underlying sialic acid as an essential nutrient for brain development and cognition. $A d v$ Nutr 2012;3:465S-472S.

5. Schnaar RL, Gerardy-Schahn R, Hildebrandt H. Sialic acids in the brain: gangliosides and polysialic acid in nervous system development, stability, disease, and regeneration. Physiol Rev 2014:94:461-518.

6. Hinderlich S, Weidemann W, Yardeni T, Horstkorte R, Huizing M. UDP-G/CNAC 2-epimerase/mannac kinase (GNE): a master regulator of sialic acid synthesis. Top Curr Chem 2015; 366:97-137.

7. Wang B, Yu B, Karim M, Hu H, Sun Y, McGreevy P, et al. Dietary sialic acid supplementation improves learning and 
memory in piglets. Am J Clin Nutr 2007;85:561-569.

8. Calandreau L, Márquez C, Bisaz R, Fantin M, Sandi C. Differential impact of polysialyltransferase ST8Siall and ST8SialV knockout on social interaction and aggression. Genes Brain Behav 2010;9:958-967.

9. Anney R, Klei L, Pinto D, Regan R, Conroy J, Magalhaes TR, et al. A genome-wide scan for common alleles affecting risk for autism. Hum Mol Genet 2010;19:4072-4082.

10. Hane M, Kitajima K, Sato C. Effects of intronic single nucleotide polymorphisms (iSNPS) of a polysialyltransferase, ST8SIA2 gene found in psychiatric disorders on its gene products. Biochem Biophys Res Commun 2016:478:11231129.

11. Toro R, Konyukh M, Delorme R, Leblond C, Chaste $\mathrm{P}$, Fauchereau $\mathrm{F}$, et al. Key role for gene dosage and synaptic homeostasis in autism spectrum disorders. Trends Genet 2010; 26:363-372.

12. Brondino N, Fusar-Poli L, Rocchetti M, Bertoglio F, Bloise N, Visai $\mathrm{L}$, et al. BDNF levels are associated with autistic traits in the general population. Psychoneuroendocrinology 2018; 89:131-133.

13. Sato C, Hane M, Kitajima K. Relationship between ST8SIA2, polysialic acid and its binding molecules, and psychiatric disorders. Biochim Biophys Acta 2016;1860:1739-1752.

14. Yang X, Liang S, Wang L, Han P, Jiang X, Wang J, et al. Sialic acid and anti-ganglioside antibody levels in children with autism spectrum disorders. Brain Res 2018;1678:273-277.

15. Kamien B, Harraway J, Lundie B, Smallhorne L, Gibbs V, Heath $\mathrm{A}$, et al. Characterization of a $520 \mathrm{~kb}$ deletion on chromosome 15q26. 1 including ST8SIA2 in a patient with behavioral disturbance, autism spectrum disorder, and epilepsy. Am J Med Genet A 2014;164A:782-788.

16. Waszkiewicz N, Chojnowska S, Zalewska A, Zwierz K, Szulc A, Szajda SD. Salivary hexosaminidase in smoking alcoholics with bad periodontal and dental states. Drug A/cohol Depend 2013;129:33-40.

17. Wang B, Miller JB, Sun Y, Ahmad Z, McVeagh P, Petocz P. A longitudinal study of salivary sialic acid in preterm infants: comparison of human milk-fed versus formula-fed infants. J Pediatr 2001;138:914-916.

18. Hartley-McAndrew M, Mertz J, Hoffman M, Crawford D. Rates of autism spectrum disorder diagnosis under the DSM-5 criteria compared to DSM-IV-TR criteria in a hospital-based clinic. Pediatr Neurol 2016;57:34-38.

19. Krug DA, Arick J, Almond P. Behavior checklist for identifying severely handicapped individuals with high levels of autistic behavior. J Child Psychol Psychiatry 1980;21:221-229.

20. Meng WD, Sun SJ, Yang J, Chu RX, Tu W, Liu Q. Elevated serum brain-derived neurotrophic factor (BDNF) but not BDNF gene Val66Met polymorphism is associated with autism spectrum disorders. Mol Neurobiol 2017;54:1167-1172.

21. Aman M, Singh N. The Aaberrant Behavior ChecklistCommunity. East Aurora, NY:Slosson Education Publications,
Inc.;1994.

22. Rutishauser U. Polysialic acid in the plasticity of the developing and adult vertebrate nervous system. Nat Rev Neurosci 2008;9:26-35.

23. Bork K, Reutter W, Gerardy-Schahn R, Horstkorte R. The intracellular concentration of sialic acid regulates the polysialylation of the neural cell adhesion molecule. FEBS Lett 2005;579:5079-5083.

24. Sgadò P, Genovesi S, Kalinovsky A, Zunino G, Macchi F, Allegra $\mathrm{M}$, et al. Loss of GABAergic neurons in the hippocampus and cerebral cortex of Engrailed-2 null mutant mice: implications for autism spectrum disorders. Exp Neurol 2013; 247:496-505.

25. Vogt D, Cho KKA, Lee AT, Sohal VS, Rubenstein JLR. The parvalbumin/somatostatin ratio is increased in Pten mutant mice and by human PTEN ASD alleles. Cell Rep 2015;11:944-956.

26. Coghlan S, Horder J, Inkster B, Mendez MA, Murphy DG, Nutt DJ. GABA system dysfunction in autism and related disorders: from synapse to symptoms. Neurosci Biobehav Rev 2012;36: 2044-2055.

27. Chao HT, Chen H, Samaco RC, Xue M, Chahrour M, Yoo J, et al. Dysfunction in GABA signalling mediates autism-like stereotypies and Rett syndrome phenotypes. Nature 2010;468: 263-269.

28. Harris AD, Singer HS, Horska A, Kline T, Ryan M, Edden RA, et al. GABA and g/utamate in children with primary complex motor stereotypies: an 1H-MRS study at 7T. AJNR Am J Neuroradiol 2016;37:552-557.

29. Pavăl D. A dopamine hypothesis of autism spectrum disorder. Dev Neurosci 2017;39:355-360.

30. Turner CA, Lewis MH, King MA. Environmental enrichment: effects on stereotyped behavior and dendritic morphology. Dev Psychobiol 2003;43:20-27.

31. Mudd AT, Fleming SA, Labhart B, Chichlowski M, Berg BM, Donovan SM, et al. Dietary sialyllactose influences sialic acid concentrations in the prefrontal cortex and magnetic resonance imaging measures in corpus callosum of young pigs. Nutrients 2017;9:1297.

32. Chen Y, Zheng Z, Zhu X, Shi Y, Tian D, Zhao F, et al. Lactoferrin promotes early neurodevelopment and cognition in postnatal piglets by upregulating the BDNF signaling pathway and polysialylation. Mol Neurobiol 2015;52:256-269.

33. Yang C, Zhu X, Liu N, Chen Y, Gan H, Troy FA 2nd, et al. Lactoferrin up-regulates intestinal gene expression of brainderived neurotrophic factors BDNF, UCHL1 and alkaline phosphatase activity to alleviate early weaning diarrhea in postnatal pig/ets. J Nutr Biochem 2014;25:834-842.

34. Al-Farsi YM, Al-Sharbati MM, Waly MI, Al-Farsi OA, Al-Shafaee MA, Al-Khaduri MM, et al. Effect of suboptimal breast-feeding on occurrence of autism: a case-control study. Nutrition 2012;28:e27-e32.

35. Shafai T, Mustafa M, Hild T, Mulari J, Curtis A. The association of early weaning and formula feeding with autism spectrum 
disorders. Breastfeed Med 2014;9:275-276.

36. Wang B, McVeagh P, Petocz P, Brand-Miller J. Brain ganglioside and glycoprotein sialic acid in breastfed compared with formula-fed infants. Am J Clin Nutr 2003;78:1024-1029.
37. Saad K, Eltayeb AA, Mohamad IL, Al-Atram AA, Elserogy Y, Bjørklund $\mathrm{G}$, et al. A randomized, placebo-controlled trial of digestive enzymes in children with autism spectrum disorders. Clin Psychopharmacol Neurosci 2015;13:188-193. 